is naturaleza 
Para la publicación de este número se ha contado con la ayuda

financiera de las siguientes instituciones:

Departamento de Filosofía y Lógica y Filosofía de la Ciencia de la Universidad de Sevilla

Facultad de Filosofía de la Universidad de Sevilla 


\section{NATURALEZA Y LIBERTAD}

Revista de estudios interdisciplinares

Número 4

Málaga, 2014

Esta revista es accesible on-line en el siguiente portal:

http://grupo.us.es/naturalezayl 


\section{Naturaleza y Libertad}

Revista de estudios interdisciplinares

Número 4

ISSN: 2254-96682014

Directores: Juan Arana, Universidad de Sevilla; Juan José Padial, Universidad de Málaga; Francisco Rodríguez Valls, Universidad de Sevilla.

Secretaria: Avelina Cecilia Lafuente, Universidad de Sevilla.

Consejo de Redacción: Jesús Fernández Muñoz, Universidad de Sevilla; José Luis González Quirós, Universidad Juan Carlos I, Madrid; Francisco Soler, Universität Dortmund / Universidad de Sevilla; Pedro Jesús Teruel, Universidad de Valencia; Héctor Velázquez, Universidad Panamericana, México.

Adjunto a la redacción: Miguel Palomo, Universidad de Sevilla

Consejo Editorial: Mariano Álvarez, Real Academia de Ciencia Morales y Políticas; Allan Franklin, University of Colorado; Michael Heller, Universidad Pontificia de Cracovia; Manfred Stöcker, Universität Bremen; William Stoeger, University of Arizona.

Consejo Asesor: Rafael Andrés Alemañ Berenguer. Universidad de Alicante; Juan Ramón Álvarez, Universidad de León; Luciano Espinosa, Universidad de Salamanca; Miguel Espinoza, Université de Strasbourg; Juan A. García González, Universidad de Málaga; José Manuel Giménez Amaya, Universidad de Navarra; Karim Gherab Martín, Urbana University, Illinois; Martín López Corredoira, Instituto de Astrofisica de Canarias; Alfredo Marcos, Universidad de Valladolid; Marta Mendonça, Universidade Nova de Lisboa; Javier Monserrat, Universidad Autónoma de Madrid; Leopoldo Prieto, Colegio Mayor San Pablo, Madrid; Ana Rioja, Universidad Complutense, Madrid. Madrid; José Luis González Recio, Universidad Complutense, Madrid; Javier Serrano, TEC Monterrey (México); Hugo Viciana, Université Paris I; Claudia Vanney, Universidad Austral, Buenos Aires; José Domingo Vilaplana, Huelva.

\section{Redacción y Secretaria:}

Naturaleza y Libertad. Revista de estudios interdisciplinares. Departamento de Filosofia y Lógica. Calle Camilo José Cela s.n. E-41018 Sevilla.

Depósito Legal: MA2112-2012

욜 954.55.77.57 Fax: 954.55.16.78. E-mail: jarana@us.es

(C) Naturaleza y Libertad. Revista de Filosofía, 2014 


\section{ÍNDICE}

\section{ESTUDIOS}

Manuel Alfonseca (Universidad Autónoma de Madrid), Diseño inteligente, evolución al azar, o evolución providencial....... ..11

Paul Gilbert, S J (U. Gregoriana, Roma), Deseo de conocer ..............................................27

Javier Hernández-Pacheco (U. de Sevilla), Evolución, erotismo y origen de las especies. De vuelta desde Darwin a Platón y Aristóteles.

Miguel Ángel Herrero (U. Politécnica de Madrid), La formación de los conceptos científicos. De Grosseteste a Galileo.

Ma Teresa Pelacho López (Zaragoza), Otra autonomía de la ciencia. Reflexiones a la luz del pensamiento de Serguei Kara-murzá.......

Juan Fernando Sellés (U. de Navarra), Estudio de las tesis centrales de Millán-Puelles sobre la libertad....... 189

Francisco Soler Gil (U. de Sevilla), El naturalismo y la tentación de las extrapolaciones omnicomprensivas.

Héctor Velázquez Fernández (U. Panamericana, México), En torno a la naturalización de la conciencia sugerida por Daniel Dennett

\section{SECCIÓN BIBLIOGRÁFICA}

Maria Camila Gallego: Falsos saberes.

Juan Arana: Bernardino Telesio, La naturaleza según sus propios principios. 


\title{
EL NATURALISMO Y LA TENTACIÓN DE LAS EXTRAPOLACIONES OMNICOMPRENSIVAS
}

\author{
Francisco Soler Gil. Universidad de Sevilla
}

\begin{abstract}
Resumen: Se suele argumentar que el naturalismo, al menos el metodológico, es la actitud natural del científico. En este artículo argumento que esta afirmación es cuestionable, puesto que el naturalista, al dar por supuesta la correspondencia exacta entre la realidad y el objeto de las ciencias naturales, tiende a provocar al menos dos tipos de desarrollos problemáticos: (1) en primer lugar, la generación y consolidación de pseudoexplicaciones en los temas en los que un programa científico encuentra una resistencia severa; (2) y en segundo lugar, la puesta en entredicho de lo que no son objetos sino presupuestos de la investigación científica.

Palabras clave: Naturalismo, método científico, ciencias naturales, actitud natural del científico, Thomas Nagel.

Abstract: It is often argued that naturalism (at least methodological naturalism) is the natural attitude of the scientist. In this argument article I argue that this assertion is questionable, since the naturalist, by taking for granted the exact correspondence between reality and the object of the natural sciences, tends to cause at least two types of problematic developments: (1) first the generation and consolidation of pseudo-explanations on issues where a scientific program finds severe resistance; (2) and second, the questioning of basic assumptions of scientific research.
\end{abstract}

Keywords: Naturalism, scientific method, science, natural attitude of the scientist, Thomas Nagel.

Recibido: 03/03/2014. Aprobado: 21/03/2014 
Naturaleza y Libertad. Revista de estudios interdisciplinares. Número 4, 2014. ISSN: 2254-9668

\section{INTRODUCCIÓN: ACERCA DE LA DEFINICIÓN DE «NATURALISMO»}

En este artículo pretendo ocuparme de la corriente dentro del pensamiento filosófico actual que suele denominarse «naturalista». Para ello, para que lo más apropiado sería comenzar precisando la noción de «naturalismo», tal y como la entienden los partidarios de esta corriente, y como se emplea en las discusiones actuales.

Sin embargo, si la empresa de definir cualquier concepto filosófico suele resultar complicada, la de definir el naturalismo, de un modo que haga justicia a la posición real de sus distintos representantes, nos sitúa ante un auténtico galimatías. Tanto más sorprendente cuanto que no nos estamos refiriendo a un concepto muy viejo, sino a una corriente filosófica que empieza a ser pujante en los últimos dos siglos. Por eso, el conocido estudio sobre el naturalismo editado hace una década en Alemania por Keil y Schnädelbach, avisaba ya en el primer párrafo de lo siguiente:

Las presentaciones programáticas de los naturalistas adoptan con frecuencia la forma de declaraciones generales de solidaridad con las ciencias naturales, que uno no puede contradecir razonablemente. Pero si uno intenta comprometer al naturalista con tesis identificables, más allá de esas declaraciones de solidaridad, se gana uno con facilidad el reproche de estar atribuyéndole algo que él, como naturalista, no tiene por qué afirmar. Los debates entre naturalistas y antinaturalistas declarados desembocan con frecuencia en intentos de probar que el objetivo de las críticas de la parte contraria es un hombre de paja (Keil y Schnädelbach, 2000: 7).

Empiezo advirtiendo de este peligro porque sin duda acecha al resto de lo que voy a comentar en este artículo. 
No obstante, por alguna definición hay que comenzar, por muy insuficiente que resulte para hacerse cargo de todas las voces en disputa. De manera que propongo tomar como punto de partida la noción que se expone en el primer párrafo del artículo de David Papineau sobre el «naturalismo», en la Enciclopedia Stanford de Filosofía. Es una elección pragmática, basada en la popularidad que actualmente goza esa enciclopedia entre los filósofos. Según Papineau, el naturalista es un pensador que se compromete con dos tesis:

[La] realidad se agota en la naturaleza, sin contener nada "sobrenatural", y [...] el método científico debe ser usado para investigar todos los aspectos de la realidad, incluyendo el “espíritu humano” (Papineau, 2007:1)

Puede resultar a primera vista llamativo que lo primero que se indique como característico del naturalismo sea la exclusión de lo «sobrenatural». Sin embargo, hay que decir en honor de Papineau, que su tesis no hace más que reflejar fielmente una preocupación básica habitual entre los pensadores naturalistas. Si se le pregunta a un naturalista qué entiende por naturaleza, se le está poniendo frente a una cuestión problemática, que está en la raíz de muchas de las divisiones internas de esta corriente. (¿Hay que considerar los objetos matemáticos en general, o los números naturales en particular, como realidades naturales? ¿Hay que considerar los datos subjetivos, como por ejemplo los «qualia», como realidades naturales? ¿Y lo que en física se denominan leyes y principios: son algo real o no?) Sin embargo, se nos dirá, aunque no esté claro si debemos considerar muchas entidades de diferentes clases como parte del mobiliario de lo real - es decir, como parte de la $\mathrm{Na}-$ 
turaleza - en algunos aspectos la distinción es nítida: Dios y los ángeles, por ejemplo, no son realidades naturales. Luego no existen.

Con todo, resulta un tanto insatisfactorio conformarse con un planteamiento que diga algo así como: La realidad se identifica con la Naturaleza, que es algo que no podemos definir bien, pero que excluye en todo caso a Dios.

Por eso, la mayor parte de los naturalistas tienden a precisar de un modo u otro el ámbito de lo natural. Y aunque los distintos autores discrepan en sus propuestas, lo estándar es el intento de definir ese ámbito recurriendo a las ciencias naturales.

Y eso explica la segunda tesis en la definición de Papineau: «el método científico debe ser usado para investigar todos los aspectos de la realidad». Que puede reformularse también así: La realidad —es decir, la Naturalezacoincide con el ámbito de lo investigable siguiendo el método científico.

Por supuesto, este planteamiento da lugar a nuevas preguntas que introducen nuevos problemas. Por ejemplo: ¿Queda determinado el ámbito de lo real por el alcance de las ciencias naturales en su estado actual? ¿O más bien por lo que «potencialmente» podrían llegar a conocer las ciencias naturales? ¿Y qué ciencias merecen el calificativo de «naturales»? ¿También las denominadas «ciencias humanas», o las «ciencias sociales»? ¿O sólo la física, en el fondo? ¿Tiene que ofrecer el naturalismo prescripciones metodológicas que determinen qué procedimientos pueden considerarse propios de la ciencia natural? Y, ¿tiene que comprometerse el naturalismo con alguna empresa de unificación de las ciencias naturales, o no?

Dejemos abiertas todas estas preguntas y retengamos, para el resto del artículo, tan sólo la idea general de que el naturalista, por lo común, establece 228 
una identificación entre lo real y lo accesible a las disciplinas que sean, o puedan ser, reconocidas como científicas.

Adicionalmente, podemos apuntar aquí la distinción entre el «naturalismo ontológico», que afirmaría que lo real coincide con lo que se encuentra al alcance (si quiera potencial) de alguna ciencia natural, y el «naturalismo metodológico», más débil si se quiere, que se limitaría a afirmar que en la investigación científica debemos proceder como si aceptáramos esa identificación entre lo real y lo accesible al método científico. Sea la realidad así, o no lo sea, la forma de hacer buena ciencia es trabajando como si lo fuera.

Bien. Estoy seguro de que a estas alturas del discurso ya habrá algún naturalista dispuesto a alzar su voz para acusarme de haber construido un hombre de paja. Pero creo que, excepciones aparte, lo esbozado responde con fidelidad a las ideas más usuales en esta corriente. De manera que voy a tomar lo dicho hasta ahora como punto de partida para exponer, en el resto del artículo, una duda — nada más que eso: una duda — sobre la idoneidad de la perspectiva naturalista como acompañante de la actividad científica. Veamos de qué se trata.

\section{LA TENTACIÓN DE LAS EXTRAPOLACIONES OMNICOMPRENSIVAS}

En su obra «Sucesivos escolios a un texto implícito», el pensador colombiano Nicolás Gómez Dávila incluyó el siguiente aforismo:

Después de resolver un problema, la humanidad se imagina hallar en soluciones análogas la clave de todos.

Toda solución auténtica arrastra un cortejo de soluciones grotescas (Gómez Dávila, 2002:44). 
En este aforismo se encuentra condensado, en su mínima expresión, una forma de paso en falso que, a mi modo de ver, podría estar ligado esencialmente al programa naturalista. Es lo que cabe denominar la «tentación de las extrapolaciones omnicomprensivas». Esta tentación, surgiría al dar por supuesta la correspondencia exacta entre la realidad y el objeto de las ciencias naturales, y tendería a provocar al menos dos tipos de desarrollos problemáticos: (1) en primer lugar, la generación y consolidación de pseudoexplicaciones en los temas en los que un programa científico encuentra una resistencia severa; (2) y en segundo lugar, la puesta en entredicho de lo que no son objetos sino presupuestos de la investigación científica.

En esta ponencia me voy a centrar, por cuestión de espacio, en el primero de estos problemas. Pero antes de entrar en él, permítaseme apuntar, con toda brevedad, algo sobre el segundo:

$\mathrm{Si}$, como nos dice Papineau, el método científico debe ser usado para investigar todos los aspectos de la realidad, ¿qué hacemos entonces con lo que no es objeto sino presupuesto de las ciencias? ¿Qué estatuto le quedará a todo lo que no puede ser entendido por medio de extrapolaciones de estrategias científicas exitosas porque es previo a esas estrategias?

Me refiero, por ejemplo, a la propia racionalidad de la naturaleza, y también a la capacidad humana para comprender la racionalidad natural (es decir, la tesis de la correspondencia entre razón objetiva y razón subjetiva). Las ciencias no llegan a estas tesis como resultado de una investigación específica que las tenga por objeto. Más bien constituyen supuestos tácitos de la empresa científica. Supuestos que, en todo caso, se vuelven más verosímiles cuantos más éxitos particulares se logren en la empresa científica. Pero su- 
puestos que, por sí mismos, no pueden ser enfocados como objeto al que se le puedan aplicar los métodos de estudios establecidos por ninguna ciencia. Porque cualquier método que tratáramos de aplicar para estudiarlos científicamente estaría presuponiendo de entrada su validez.

En consecuencia, como no pueden constituir el objeto de ninguna ciencia, su estudio debe ser rechazado, considerado como una ilegítima maniobra metafísica. Pero entonces desembocamos en que las ciencias naturales, que son la medida de todas las cosas, dependen de postulados que no pueden ser justificados como especificaciones de aspectos de la realidad, según el criterio de Papineau. De modo que es, por así decirlo, como si la ciencia se sostuviera sobre el vacío. Quiero decir, sobre un conjunto de opciones arbitrarias.

Este es un problema serio, que se deriva de extrapolar los procedimientos que nos han proporcionado conocimientos parciales (en física, en biología, etc.), hasta exigir que todo lo real sea sometible a ellos. Pero no voy a desarrollarlo aquí con más extensión.

En lugar de eso, propongo que prestemos atención a esta otra dificultad:

Habíamos quedado en que, si damos por supuesta la correspondencia exacta entre la realidad y el objeto de las ciencias naturales, entonces todos los fenómenos reales deben tener su explicación en el marco de las teorías científicas. En principio, se podría advertir (curándonos en salud) que esa correspondencia sólo debe darse con unas teorías científicas ideales, situadas al término de un proceso (quizás) infinito de progreso científico. Pero, en la práctica, las teorías científicas son las que son, las que se encuentran al presente respaldadas de modo estándar por la comunidad científica. Y si estas teorías no pudieran dar cuenta de aspectos importantes de lo real, y si no se percibieran avances significativos en la incorporación de esos aspectos de la 
realidad al marco de lo explicado por las ciencias, podría surgir la duda sobre el programa naturalista. Por eso, si un científico o filósofo de la naturaleza (o filósofo de la ciencia) acepta la perspectiva naturalista, se encuentra por fuerza sometido a una fuerte presión mental tendente a la aceptación de «soluciones grotescas», por usar la terminología de Gómez Dávila, allá donde las soluciones auténticas ya no alcanzan. Es decir, la tentación de extrapolar a toda costa las estrategias que han proporcionado determinados avances en la ciencia a cualquier tipo de temas adyacentes. Más aún, se encuentra sometido a una fuerte presión defensiva de esas pseudoexplicaciones, que pueden llegar a enmascarar la falta real de explicación, y convertir la ciencia en una fabricadora de extrapolaciones omnicomprensivas, cada vez más lejos de la verdad.

Bien. Esto que he descrito es una posibilidad teórica. La pregunta ahora es, ¿̇tenemos algún indicio de que este tipo de desarrollos problemáticos están realmente ocurriendo? A mi modo de ver sí tenemos indicios en este sentido, sobre todo relacionados con las explicaciones de lo mental.

Un ejemplo muy significativo lo proporcionan las reacciones a que ha dado lugar la reciente aparición (en 2012) del libro de Thomas Nagel «Mind and cosmos». Esta obra de Nagel — que lleva el significativo título de «Por qué la concepción materialista neodarwiniana de la naturaleza es falsa casi con seguridad»—, tiene, como ocurre con todos los libros, puntos más fuertes que otros, y tesis más discutibles que otras. En todo caso, su objetivo es criticar lo que Nagel denomina

una exhaustiva y especulativa imagen del mundo, alcanzada por medio de la extrapolación de ciertos descubrimientos de la biología, la química y la física; una imagen del mundo naturalista particular, que postula una relación jerárquica entre los sujetos de dichas cien- 
cias, y la completitud en principio de una explicación de todo en el universo por medio de su unificación (Nagel, 2012: 4)

Y el gran motor de la crítica, que Nagel deriva de su larga trayectoria de estudio de los problemas de la filosofía de la mente, es su afirmación de que no podemos explicar realmente la consciencia y sus fenómenos por medio de la base ontológica que nos proporciona la física, la química y la biología. Es decir, no podemos explicarla por medio de las extrapolaciones reduccionistas que guían las actuales aproximaciones al tema desde la perspectiva evolucionista y de las neurociencias.

No puedo entrar, en el espacio de este artículo, a resumir sus argumentos en favor de esta tesis ${ }^{1}$, ni cómo Nagel va derivando luego de ella la radical incompletitud de la descripción de la naturaleza que nos proporcionan todas las ciencias naturales actuales. En todo caso, para las reflexiones que estoy tratando de presentar aquí, resultan casi tan significativas las reacciones pro-

1 Baste mencionar que el núcleo del planteamiento de Nagel es que: «Los materialistas tendrían que explicar cómo „dolor“ y „estado cerebral“ pueden referirse a la misma cosa a pesar de que su significado no es el mismo, y explicar esto sin apelar a algo no físico para dar cuenta de la referencia de „dolor“. Estas estrategias han tomado una forma cada vez más sofisticada, bajo los títulos de conductismo causal, funcionalismo y otras teorías de cómo los conceptos mentales podrían referir a estados del cerebro en virtud del papel causal de esos estados en el control de la interacción entre el organismo y su entorno. Pero todas estas estrategias resultan insatisfactorias por la misma vieja razón: incluso añadiendo el cerebro a la pintura, dejan claramente fuera algo esencial, sin lo cual no habría mente. Y lo que dejan fuera es justo aquello que fue dejado deliberadamente fuera del mundo físico por Descartes y Galileo en orden a constituir el concepto moderno de lo físico, a saber, las apariencias subjetivas» (Nagel, 2012: 39-40). 
vocadas por el libro de Nagel como el propio libro. Desde hace meses vengo siguiendo las recensiones de esta obra que se publican, y no he podido menos que sorprenderme por el tono agrio, y con frecuencia sazonado de descalificaciones «ad hominem» de las recensiones escritas por autores identificados con el proyecto explicativo criticado por Nagel. No voy a traer citas literales de este tipo de materiales, porque me parece que no son propias de una discusión filosófica. Pero sí me gustaría apuntar que esta acritud podría ser una muestra de esa presión mental — que mencionaba anteriormente - tendente a la aceptación y mantenimiento de cualquier propuesta explicativa, por deficiente que sea, que permita incluir todos los elementos reconocidos de lo real dentro del marco definido por las teorías que tenemos a mano. Una presión propia de haber adoptado el enfoque naturalista.

Si voy a mencionar la recensión del conocido biólogo H. Allen Orr, publicada en The New York Review of Books. Pues, pese a la escasa simpatía de Orr por el planteamiento de Nagel, me parece un ejemplo de análisis equilibrado y desapasionado que resulta poco frecuente y muy de agradecer. Critica Orr, a mi entender con toda razón, los argumentos de Nagel tendentes a arrojar dudas sobre las explicaciones neodarwinistas del proceso evolutivo. Pero luego añade lo siguiente:

\footnotetext{
Mientras que las preocupaciones de Nagel respecto al neodarwinismo están fuera de lugar, cuando se vuelve a los fenómenos mentales como la consciencia se encuentra en un terreno algo más firme (o al menos más familiar). Después de todo, estos son problemas distintos. Una ciencia podría explicar la evolución de la vida, pero dejar la consciencia -la experiencia subjetiva de lo salado de las palomitas, del choque del agua fría, o del aguijón del dolor- sin contabilizar (Orr, 2013: 6)
} 
Tras reconocer el interés del análisis de Nagel del problema de las explicaciones al uso de la consciencia, Orr aportará luego dos argumentos que podrían llevarnos en otra dirección (el primero: que la historia de la ciencia muestra que muchos obstáculos terminan siendo removidos; el segundo: que quizás la consciencia no pueda ser comprendida simplemente como consecuencia de las limitaciones cognitivas de una mente surgida en el proceso evolutivo).

No voy a entrar en estas cuestiones, que nos desviarían del tema que estoy tratando de enfocar. Pero sí quiero volver a llamar la atención sobre el escenario que Orr plantea en la cita anterior: La teoría neodarwinista de la evolución podría ser correcta, pero no tener nada que decir sobre algo como la consciencia, o como lo mental en general.

Este escenario no parece problemático si uno considera que las teorías científicas son proyecciones parciales de una realidad más rica, que no pueden agotar exhaustivamente. (Un planteamiento, que, dicho sea de paso, han compartido la mayor parte de los científicos más creativos a lo largo de la historia de las ciencias modernas). Pero en cambio, si nos situamos en la correspondencia naturalista entre realidad y objeto de las ciencias naturales, semejante propuesta resulta muy difícil de aceptar. Pues la consciencia parecería quedar al margen de la red de explicaciones científicas que componen la Naturaleza. De ahí la tendencia a considerar la consciencia explicada, o en trance de ser explicada, desde la biología actual. [Véase, por ejemplo, las obras de Dennett sobre la consciencia (Dennett, 1991), la libertad (Dennett 2004), y otros asuntos problemáticos: Quizás las obras de Dennett representen el mejor ejemplo de lo que estoy tratando de caracterizar como la «tentación de las extrapolaciones omnicomprensivas»]. 
Por último, me gustaría concluir este punto del argumento subrayando que el libro de Nagel, no ha sido, ni mucho menos, la única llamada de atención sobre las deficiencias de las «explicaciones» de lo mental que se están realizando desde las ciencias naturales actuales. Por falta de espacio, no puedo entrar en otras aportaciones, como, por ejemplo, el muy interesante libro «El mito del determinismo», de Brigitte Falkenburg, que contiene, entre otras cosas, una crítica argumentada con gran detalle de los intentos reductores de lo mental apoyados en las neurociencias.

Estas críticas podrán resultar a la postre correctas o no. Pero apuntan en todo caso a un campo en el que la adopción de la perspectiva naturalista nos fuerza a aceptar extrapolaciones (como poco) arriesgadas, al tiempo que nos impulsa a negar dificultades que podrían ser muy reales.

La tentación de las extrapolaciones omnicomprensivas constituye un peligro innegable que acecha al que se propone el estudio de ciertos temas, partiendo de una perspectiva naturalista. Advertido lo cual, podemos pasar a considerar (muy brevemente) el último punto del que quería ocuparme en este artículo.

\section{3. ¿LA ACTITUD NATURAL DEL CIENTÍFICO?}

Se suele argumentar que el naturalismo, al menos el metodológico, es la actitud natural del científico. Quizás incluso la única actitud que puede adoptar, o la que adopta siempre de forma instintiva. Y que esto es así porque, de otro modo, ante cualquier dificultad explicativa, bastaría con recurrir a la voluntad de un «Dios de los huecos», para quitárnosla de enmedio, con una pseudoexplicación, que ocultaría la necesidad de una explicación real. 
Sin embargo, si la argumentación que he venido desarrollando en esta ponencia es correcta, la perspectiva naturalista nos sitúa ante una tentación muy parecida: la de aceptar, a falta de algo mejor, cualquier explicación que se obtenga extrapolando sobre la base de las teorías científicas reconocidas en el momento, aunque sea a costa de simplemente tapar los problemas reales bajo un análisis que niega la existencia de los datos, o los aspectos, que no pueda derivar de la base explicativa aceptada.

Si esto es así, el naturalismo constituiría un pensamiento de fondo tan poco recomendable al científico como la creencia en la magia.

Ahora bien, ¿qué actitud sería recomendable entonces al estudioso de la naturaleza? ¿Cuál tendría que ser la «actitud natural» del científico?

Bueno, quizás no exista algo así como una «actitud natural». Cada científico tiene su forma de pensar la ciencia, como herramienta más o menos poderosa, más o menos completa o limitada, etc. Y, al final, las aportaciones positivas de los grandes investigadores pueden ser bastante independientes de lo que piensen sobre estos asuntos.

Pero, si se me obligara a definir una «actitud natural» del científico, apuntaría justo a la que he mencionado antes, al hilo de la cita de Orr, y que, a mi modo de ver, ha sido común a la mayor parte de los científicos a lo largo de la historia: El convencimiento de que nuestras teorías científicas no son más que proyecciones de una realidad que seguramente no agotan. Proyecciones cuyo poder explicativo es bueno desarrollar al máximo de sus posibilidades, pero sin convertir la posibilidad de explicarlo todo con ellas en una cuestión de principio. La disposición a llevar esas proyecciones hasta donde se vea que funcionan bien, pero sin precipitarse a extenderlas donde hay serios motivos para sospechar que ya no dan más de sí. La actitud, en 
Naturaleza y Libertad. Revista de estudios interdisciplinares. Número 4, 2014. ISSN: 2254-9668

definitiva, de conjugar la curiosidad científica con el convencimiento de que la realidad es más grande que los modelos que nos hacemos de ella.

\section{BIBLIOGRAFÍA}

D. Dennett, La Conciencia Explicada, Barcelona, Paidós, 1991.

—, La Evolución de la Libertad, Barcelona, Paidós, 2004.

B. Falkenburg, Mythos Determinismus, Berlin, Springer, 2012.

N. Gómez Dávila, Sucesivos Escolios a un Texto Implícito, Barcelona, Áltera, 2002.

G. Keil y H. Schnädelbach, Naturalismus, Frankfurt, Suhrkamp, 2000.

T. Nagel, Mind and Cosmos, Oxford, Oxford University Press, 2012.

H. A. Orr, «Awaiting a new Darwin», en: The New York Review of Books 7 de febrero 2013.

D. Papineau, «Naturalism», Stanford Encyclopaedia of Philosophy, 2007. Disponible en: http://plato.stanford.edu/entries/naturalism/

Francisco Soler Gil

soler@unibremen.de 